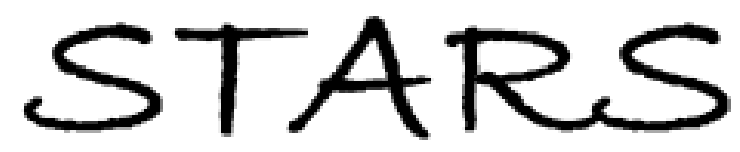

University of Central Florida

STARS

$1-1-1985$

\title{
Relativistic Modulational Instability Of An Electromagnetic Wave In A Magnetized Plasma
}

Bhimsen K. Shivamoggi

University of Central Florida

Find similar works at: https://stars.library.ucf.edu/facultybib1980

University of Central Florida Libraries http://library.ucf.edu

This Article is brought to you for free and open access by the Faculty Bibliography at STARS. It has been accepted for inclusion in Faculty Bibliography 1980 s by an authorized administrator of STARS. For more information, please contact STARS@ucf.edu.

\section{Recommended Citation}

Shivamoggi, Bhimsen K., "Relativistic Modulational Instability Of An Electromagnetic Wave In A Magnetized Plasma" (1985). Faculty Bibliography 1980s. 446.

https://stars.library.ucf.edu/facultybib1980/446

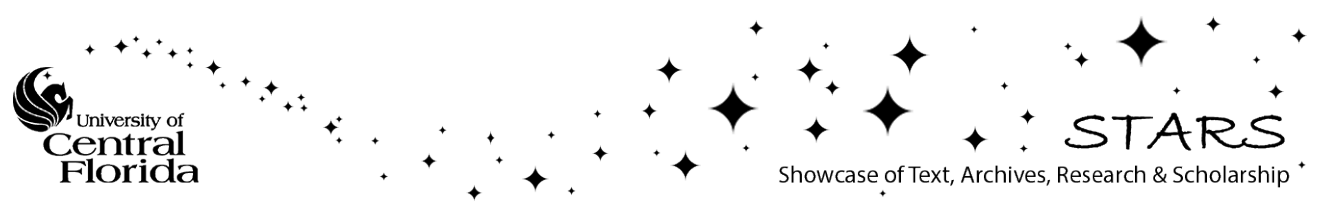




\title{
Relativistic modulational instability of an electromagnetic wave in a magnetized plasma
}

\author{
Bhimsen K. Shivamoggi* \\ Department of Mathematics, University of Central Florida, Orlando, Florida 32816
}

(Received 15 November 1984)

\begin{abstract}
The relativistic modulational instability of an intense circularly polarized electromagnetic wave propagating along an external magnetic field in a plasma is investigated. The method of investigation is based on the derivation of a nonlinear Schrödinger equation. The effect of the external magnetic field is to diminish the modulational instability of the circularly polarized wave. Actually, the modulational instability can be completely eliminated if the external magnetic field is sufficiently strong.
\end{abstract}

\section{INTRODUCTION}

Nonlinear interaction of very intense laser pulses with plasma is of great interest in laboratory plasmas. At very high laser intensities of current interest, the directed velocity of the electrons become relativistic. The consequent electron-mass variation can lead to modulational instability of electromagnetic waves propagating through the plasma (Max, Arons, and Langdon ${ }^{1}$ ). The latter enables the incident laser pulse to propagate into denser regions of the plasma, and therefore, plays an important role in transferring the laser energy to the plasma. The usual ponderomotive-force driven modulational instabilities (Drake et al. ${ }^{2}$ and Kaw, Schmidt, and Wilcox ${ }^{3}$ ) involving ion motions occur with characteristic frequencies $\mid \omega_{\mid}^{2} \leq \omega_{p_{i}}^{2}$. However, the relativistic modulational instability arising with high laser intensities involves only electron motions. This is because this instability can grow so quickly in time that ion inertia prevents the ions from following along.

Experiments of Stamper et al. ${ }^{4}$ and some others showed that intense spontaneously generated magnetic fields are present in laser-produced plasmas. Therefore, some studies have been made of the laser interaction in a magnetized plasma (Aliev and Kuznetsov, ${ }^{5}$ Berezhiani, Paverman, and Tskhakaya, ${ }^{6}$ and Rao, Shukla, and $\mathrm{Yu}^{7}$ ). We investigate here the modulational instability of an intense circularly polarized electromagnetic wave propagating along a constant external magnetic field in the plasma. The method of investigation is different from the one used by Max et al., ${ }^{1}$ and is based on the derivation of a nonlinear Schrödinger equation.

\section{GOVERNING EQUATIONS}

Consider the propagation of an intense circularly polarized electromagnetic wave of frequency $\omega_{0}$ and wave number $k_{0}$ in a plasma subjected to a state external magnetic field $\mathbf{B}_{0}$. In the modulational interaction problem the plasma response is two-pronged: the high-frequency motion of the electrons in the wave field, and the lowfrequency motion caused by the ponderomotive force exerted by the self-interaction of the wave. The relativistic modulational processes that occur in cases involving high laser intensities occur at a rate too rapid for the ions to participate in them. Therefore, we consider the ions to remain immobile and to form a neutralizing background, and consider only the electron response. Further, we shall neglect the thermal motion of the electrons in comparison with the directed motion. We then have the following equations governing the motion of the electron fluid:

$$
\begin{aligned}
& \frac{\partial n}{\partial t}+\nabla \cdot(n \mathbf{V})=0, \\
& \frac{\partial \mathbf{p}}{\partial t}+(\mathbf{V} \cdot \boldsymbol{\nabla}) \mathbf{p}=-e\left[\mathbf{E}+\frac{1}{c} \mathbf{V} \times\left(\mathbf{B}_{0}+\mathbf{B}\right)\right], \\
& \boldsymbol{\nabla} \times \mathbf{E}=-\frac{1}{c} \frac{\partial \mathbf{B}}{\partial t}, \\
& \boldsymbol{\nabla} \times \mathbf{B}=-\frac{4 \pi n e}{c} \mathbf{V}+\frac{1}{c} \frac{\partial \mathbf{E}}{\partial t}, \\
& \nabla \cdot \mathbf{E}=-4 \pi e\left(n-n_{0}\right), \\
& \boldsymbol{\nabla} \cdot \mathbf{B}=0,
\end{aligned}
$$

where $n$ is the number density, $\mathbf{V}$ is the velocity of the fluid, $c$ is the speed of light,

$$
\mathbf{p}=\frac{m_{0} \mathbf{V}}{\left(1-V^{2} / c^{2}\right)^{1 / 2}},
$$

$m_{0}$ is the rest mass of an electron, and $n_{0}$ is the unperturbed number density. Let us introduce the scalar and vector potentials $\phi$ and $\mathbf{A}$ to describe the wave fields $\mathbf{E}$ and $\mathbf{B}$ :

$$
\mathbf{B}=\boldsymbol{\nabla} \times \mathbf{A}, \quad \mathbf{E}=-\boldsymbol{\nabla} \phi-\frac{1}{c} \frac{\partial \mathbf{A}}{\partial t} .
$$

$\phi$ turns out to be the low-frequency ambipolar potential.

Let us consider a left-hand circularly polarized wave given by

$$
\mathbf{A}=\boldsymbol{A}(z, t)\left(\hat{\mathbf{i}_{z}}-i \hat{\mathbf{i}_{y}}\right) \cos \theta, \quad \theta=k_{0} z-\omega_{0} t
$$

to propagate along $\mathbf{B}_{0}=\boldsymbol{B}_{0} \hat{\mathbf{i}_{z}}$, with the linear wave propagation relation

$$
\omega_{0}^{2}=c^{2} k_{0}^{2}+\frac{\omega_{p}^{2} \omega}{\omega+\Omega_{0}},
$$


where

$$
\omega_{p}^{2}=\frac{4 \pi n_{0} e^{2}}{m_{0}}, \Omega_{0}=\frac{e B_{0}}{m_{0} c} .
$$

Using (7) and (8), Eq. (2) can be written as

$$
\begin{aligned}
\frac{\partial \mathbf{p}}{\partial t}+ & \frac{1}{m_{0}\left(1+p^{2} / m_{0}^{2} c^{2}\right)^{1 / 2}}(\mathbf{p} \cdot \boldsymbol{\nabla}) \mathbf{p} \\
= & \frac{e}{c} \frac{\partial \mathbf{A}}{\partial t}+e \boldsymbol{\nabla} \phi \\
& -\frac{e / m_{0} c}{\left(1+p^{2} / m_{0}^{2} c^{2}\right)^{1 / 2}}\left\{\mathbf{p} \times\left[(\boldsymbol{\nabla} \times \mathbf{A})+\mathbf{B}_{0}\right]\right\}
\end{aligned}
$$

from which it follows that (Aliev et al., ${ }^{5}$ and Rao et al. ${ }^{7}$ )

$$
\begin{aligned}
& A \cos \theta=\frac{c p}{e}+\alpha \frac{c p / e}{\left(1+P^{2} / m_{0}^{2} c^{2}\right)^{1 / 2}}, \\
& \nabla \phi=c^{2} \nabla\left[\left(1+p^{2} / m_{0}^{2} c^{2}\right)^{1 / 2}-\frac{\alpha / 2}{1+p^{2} / m_{0}^{2} c^{2}}\right]=0,
\end{aligned}
$$

where

$$
\alpha \equiv \frac{\Omega_{0}}{\omega_{0}}
$$

and Eq. (13) has been obtained by ignoring the electron momentum in the wave propagation direction in the lowfrequency plasma response.

\section{NONLINEAR SCHRÖDINGER EQUATION}

We obtain from Eqs. (3), (4), and (6) the wave equation

$$
\frac{\partial^{2} \mathbf{A}}{\partial t^{2}}-c^{2} \nabla^{2} \mathbf{A}=-4 \pi n e c \mathbf{V}
$$

Using (9) and (10) and noting that $A(Z, t)$ is a slowlyvarying function of $Z$ and $t$, we obtain from Eq. (14),

$$
\begin{gathered}
{\left[i\left[\frac{\partial A}{\partial t}+\frac{k_{0} c^{2}}{\omega_{0}} \frac{\partial A}{\partial z}\right]+\frac{c^{2}}{2 \omega_{0}} \frac{\partial^{2} A}{\partial z^{2}}\right] \cos \theta} \\
\left.=\frac{\omega_{p}^{2}}{2 \omega_{0}} \int \frac{(c \mathrm{p} / e)\left(n / n_{0}\right)}{\left(1+p^{2} / m_{0}^{2} c^{2}\right)^{1 / 2}}-\frac{A \cos \theta}{1+\alpha}\right] .
\end{gathered}
$$

Here,

$$
n=n_{0}+\delta n
$$

and the density perturbation $\delta n$ can be calculated from the dynamics of the plasma motion parallel to the wave propagation direction,

$$
\begin{aligned}
& \frac{\partial \delta n}{\partial t}+n_{0} \frac{\partial \delta V}{\partial z}=0, \\
& \frac{\partial \delta V}{\partial t}=-\frac{e}{m}\left[-i \frac{V_{0-} \delta B_{+}}{c}+\delta E_{z}\right), \\
& \frac{\partial \delta E_{z}}{\partial z}=-4 \pi e \delta n,
\end{aligned}
$$

where $q_{ \pm}=q_{x} \pm i q_{y}$, and

$$
V_{0-}=-\frac{e E_{0-} e^{i \theta}}{i m_{0} \omega_{0}(1+\alpha)}, \delta B_{+}=\frac{i c k_{0}}{\omega_{0}} E_{+} e^{i \theta}
$$

Equations (17) - (20) give

$$
\frac{\delta n}{n_{0}}=-\frac{e^{2} E_{0}^{2}}{m_{0}^{2} \omega_{0}^{2} c^{2}(1+\alpha)} \frac{\omega_{0}^{2}-\omega_{p}^{2} /(1+\alpha)}{4 \omega_{0}^{2}-\omega_{p}^{2}} \cos (2 \theta)
$$

or

$$
\frac{\delta n}{n_{0}}=\frac{e^{2} A^{2}}{m_{0}^{2} c^{4}(1+\alpha)} \frac{\omega_{0}^{2}-\omega_{p}^{2} /(1+\alpha)}{4 \omega_{0}^{2}-\omega_{p}^{2}} \cos (2 \theta) .
$$

Next, assuming that $p^{2} / m_{0}^{2} c^{2} \ll 1$, we obtain from (12),

$$
p \approx \frac{e A / c}{1+\alpha} \cos \theta+\frac{\alpha}{2} \frac{e^{3} A^{2} / m_{0}^{2} c^{5}}{(1+\alpha)^{3}} \cos ^{3} \theta
$$

Using (16), (21), and (22), Eq. (15) becomes

$i\left(\frac{\partial A}{\partial t}+\frac{k_{0} c^{2}}{\omega_{0}} \frac{\partial A}{\partial z}\right)+\frac{c^{2}}{2 \omega_{0}} \frac{\partial^{2} A}{\partial z^{2}}+\frac{\omega_{p}^{2} q}{4 \omega_{0}}|A|^{2} A=0$,

where

$$
q=\frac{e^{2} / m_{0}^{2} c^{4}}{(1+\alpha)^{2}}\left[\frac{3 / 4}{1+\alpha}-\frac{\omega_{0}^{2}-\omega_{p}^{2} /(1+\alpha)}{4 \omega_{0}^{2}-\omega_{p}^{2}}\right] .
$$

For the case of an unmagnetized plasma (with $\alpha=0$, Eq. (23) can be seen to arise through the familiar nonlinear frequency shift due to the relativistic effects (Sluijter and Montgomery ${ }^{8}$ ).

Upon transferring to a frame moving with speed $k_{0} c^{2} / \omega_{0}$, Eq. (23) reduces to the standard form of the nonlinear Schrödinger equation (Hasegawa ${ }^{9}$ ):

$$
i \frac{\partial A}{\partial t}+\frac{c^{2}}{2 \omega_{0}} \frac{\partial^{2} A}{\partial \xi^{2}}+\frac{\omega_{p}^{2} q}{4 \omega_{0}}|A|^{2} A=0
$$

where

$$
\xi=z-\frac{k_{0} c^{2}}{\omega_{0}} t
$$

\section{MODULATIONAL INSTABILITY}

In order to investigate stability with respect to lowfrequency modulation, let us rewrite Eq. (25) as follows:

$$
i \frac{\partial A}{\partial t}+\frac{c^{2}}{2 \omega_{0}} \frac{\partial^{2} A}{\partial \xi^{2}}+\frac{\omega_{p}^{2} q}{4 \omega_{0}}\left(|A|^{2}-\left|A_{0}\right|^{2}\right) A=0
$$

and set (Hasegawa ${ }^{9}$ )

$$
\begin{aligned}
& A=\sqrt{P(\xi, t)} e^{i \sigma(\xi, t)}, \\
& P=P_{0}+P_{1}(\xi, t), \quad \sigma=\sigma_{1}(\xi, t)
\end{aligned}
$$

and linearize in $P_{1}, \sigma_{1}$, and consider

$$
P_{1}(\xi, t) \text { and } \sigma_{1}(\xi, t) \sim e^{i(K \xi-\Omega t)} .
$$

We then obtain

$$
\Omega^{2}=\frac{c^{2} K^{2}}{4 \omega_{0}}\left(\frac{c^{2} K^{2}}{\omega_{0}}-\frac{\omega_{p}^{2} q}{\omega_{0}}\left|A_{0}\right|^{2}\right)
$$


so that the modulational instability arises if

$$
\left|E_{0}\right|^{2}>\frac{c^{4} K^{2} \omega_{0}^{2}}{\omega_{p}^{2} q} .
$$

The maximum growth rate is given by

$$
\gamma_{\max }=\frac{\omega_{p}^{2} c^{2} q}{4 \omega_{0}^{3}}\left|E_{0}\right|^{2} \text {. }
$$

Equations (30) and (31) show that the larger the external magnetic field (so that $q$ will be smaller) the larger will be the threshold value for the wave intensity to cause modulational instability, and the smaller will be the maximum growth rate. Actually, if the external magnetic field is sufficiently strong (so that $q<0$ ), the modulational instability will not arise at all!

Physically this can be understood as follows. The modulational instability arises because the group velocity of the wave increases in the direction of the increasing wave field intensity so that there is an energy transport from the region of small field intensity to the region of large field intensity. It is seen that the effect of an external magnetic field is to diminish this trend and even reverse it if sufficiently strong.

The results corresponding to the right circularly polarized wave can be obtained simply by letting $\alpha$ be negative. Because of possible gyroresonance, however, the validity of the fluid model becomes then questionable (Dimonte, Lamb, and Morales ${ }^{10}$ ).

\section{ACKNOWLEDGMENT}

I am thankful to Dr. P. K. Shukla for providing me with details of his work prior to publication.
*Permanent address: Institute of Mathematical Sciences, Madras 113, India.

${ }^{1}$ C. E. Max, J. Arons, and A. B. Langdon, Phys. Rev. Lett. 33, 209 (1974).

${ }^{2}$ J. Drake, P. K. Kaw, Y. C. Lee, C. S. Liu, G. Schmidt, and M. N. Rosenbluth, Phys. Fluids 17, 778 (1974).

${ }^{3}$ P. K. Kaw, G. Schmidt, and T. Wilcox, Phys. Fluids 16, 1522 (1973).

${ }^{4}$ J. A. Stamper, K. Papadopoulos, R. N. Sudan, S. O. Dean, E. A. Mclean, and J. M. Dawson, Phys. Rev. Lett. 26, 1012 (1971).
5Y. M. Aliev and S. V. Kuznetsov, Sov. J. Plasma Phys. 6, 205 (1980).

${ }^{6}$ V. I. Berzhiani, V. S. Paverman, and D. D. Tskhakaya, Sov. J. Plasma Phys. 6, 445 (1980).

${ }^{7}$ N. N. Rao, P. K. Shukla, and M. Y. Yu, Phys. Fluids 27, 2664 (1984).

${ }^{8}$ F. W. Sluijter, and D. Montgomery, Phys. Fluids 8, 551 (1965).

${ }^{9}$ A. Hasegawa, Plasma Instabiilty and Nonlinear Effects (Springer, Berlin, 1975).

${ }^{10}$ G. Dimonte, B. M. Lamb, and G. J. Morales, Phys. Rev. Lett. 48, 1352 (1982). 